\title{
Magnetic helicity evolution during the solar activity cycle: Observations and dynamo theory
}

\author{
N. Kleeorin ${ }^{1}$, K. Kuzanyan ${ }^{2}$, D. Moss ${ }^{3}$, I. Rogachevskii ${ }^{1}$, D. Sokoloff ${ }^{4}$, and H. Zhang ${ }^{5}$ \\ ${ }^{1}$ Department of Mechanical Engineering, Ben-Gurion University of Negev, POB 653, 84105 Beer-Sheva, Israel \\ e-mail: nat@menix.bgu.ac.il; gary@bgumail.bgu.ac.il \\ 2 IZMIRAN, Troitsk, Moscow Region 142190, Russia \\ e-mail: kuzanyan@dnttm.ru \\ 3 Department of Mathematics, University of Manchester, Manchester M13 9PL, UK \\ e-mail: moss@maths.man.ac.uk \\ ${ }^{4}$ Department of Physics, Moscow State University, Moscow 119992, Russia \\ e-mail: sokoloff@dds.srcc.msu.su \\ 5 National Astronomical Observatories, Chinese Academy of Sciences, Beijing 100012, PR China \\ e-mail: hzhang@bao.ac.cn
}

Received 14 April 2003 / accepted 15 July 2003

\begin{abstract}
We study a simple model for the solar dynamo in the framework of the Parker migratory dynamo, with a nonlinear dynamo saturation mechanism based on magnetic helicity conservation arguments. We find a parameter range in which the model demonstrates a cyclic behaviour with properties similar to that of Parker dynamo with the simplest form of algebraic $\alpha$-quenching. We compare the nonlinear current helicity evolution in this model with data for the current helicity evolution obtained during 10 years of observations at the Huairou Solar Station of China. On one hand, our simulated data demonstrate behaviour comparable with the observed phenomenology, provided that a suitable set of governing dynamo parameters is chosen. On the other hand, the observational data are shown to be rich enough to reject some other sets of governing parameters. We conclude that, in spite of the very preliminary state of the observations and the crude nature of the model, the idea of using observational data to constrain our ideas concerning magnetic field generation in the framework of the solar dynamo appears promising.
\end{abstract}

Key words. Sun: magnetic fields

\section{Introduction}

The solar activity cycle is widely believed to be connected with dynamo action which occurs somewhere inside the solar convective zone or even in the overshoot layer. Starting from the seminal paper of Parker (1955), various dynamo models have been suggested for the solar cycle (see e.g. Rüdiger \& Brandenburg 1995; Sofia et al. 1998; Tavakol et al. 2002; Brooke et al. 2002; also Blackman \& Brandenburg 2003, whose dynamo model of solar cycle also includes magnetic helicity balance). These models exploit particular parameterizations for sources of the dynamo activity, i.e. the $\alpha$-effect, which in turn is connected with the mean hydrodynamic helicity of the convective motions, and acts in conjunction with the nonuniform rotation $\Omega$. If the dynamo action is strong enough, a dynamo wave propagating somewhere inside the convective

Send offprint requests to: I. Rogachevskii, e-mail: gary@bgumail.bgu.ac.il shell is excited. It is necessary to include some saturation mechanism to get a (quasi)stationary wave which can be compared with the observed activity cycle, instead of a dynamo wave with an exponentially growing amplitude. In principle, the phenomenology of the solar cycle can be reproduced using a very primitive $\alpha$-quenching model of dynamo saturation, with the energy of the dynamo generated magnetic field achieving approximate equipartition with the kinetic energy of the random motions.

A deeper treatment of solar dynamo saturation requires however some ideas concerning the physical processes that give rise to quenching of the generation mechanism. A scenario of dynamo saturation which is now widely discussed is connected with the concept of magnetic helicity. The point is that the weakest link in the dynamo self-excitation chain, i.e. $\alpha$, is a pseudoscalar quantity and cannot be directly connected with the magnetic energy, which is a scalar (not pseudoscalar) quantity. A magnetic helicity $\chi^{m}$ can however be introduced to 
describe the level of magnetic field mirror-asymmetry and this quantity can be associated with the magnetic part of $\alpha$, i.e. $\alpha^{m}$, which is thought to be responsible for $\alpha$-quenching.

The magnetic helicity $\chi^{m}$ is an integral of motion for the ideal MHD equation, similar to the hydrodynamic helicity which is conserved in the hydrodynamical case. During the solar activity cycle, magnetic helicity is redistributed between the large and small scale magnetic field. Based on this concept, a governing equation for $\alpha^{m}$ has been suggested (Kleeorin \& Ruzmaikin 1982; Kleeorin \& Rogachevskii 1999). Together with the mean-field dynamo equations, this equation has solutions in form of a propagating steady dynamo wave (Kleeorin et al. 1994, 1995; Covas et al. 1998; Blackman \& Brandenburg 2002).

For a long time, it was impossible to observe either the magnetic helicity $\chi^{m}$ or the hydrodynamic helicity and these values, crucial for dynamo theory, were taken from theoretical estimates only. In last decade, basic progress here has been achieved and the first observations of magnetic helicity in active regions on the solar surface have been obtained (Pevtsov et al. 1994, 1995; Zhang \& Bao 1998, 1999; Canfield \& Pevtsov 1998; Longcope et al. 1998). It is possible to some extent to isolate a latitudinal distribution of magnetic helicity averaged over a solar cycle (Zhang et al. 2002) as well as to follow the temporal evolution of magnetic helicity averaged over latitude. The obvious aim now is to confront predictions of dynamo theory concerning the latitudinal distribution of magnetic helicity and its evolution during a solar cycle with the corresponding observational data; this is the aim of the present paper. When carrying out our investigation, we take into account that the available data concerning magnetic helicity of the solar magnetic field are still quite uncertain, and it would be unrealistic to expect that more or less fine details can be isolated using this data. Correspondingly, we restrict ourself to a very crude theoretical model, that we confront with observations. Specifically, we simplify the mean-field dynamo equations at the level of the Parker migratory dynamo equations, and include the algebraic $\alpha$-quenching and the dynamic $\alpha$-quenching associated with magnetic helicity evolution as the only saturation mechanisms. Both hypotheses are obvious simplifications and there is no problem in principle in including many more realistic features into our dynamo model. However we consider that to be a topic for further work.

The other point to be clarified from the very beginning is the following. Magnetic helicity can be understood as a measure of the linkage of magnetic lines and it is necessary to reconstruct the complete 3D magnetic field structure to deduce this helicity from observations. Clearly, this is a very complicated observational problem and various intermediate quantities such as current helicity $\langle\boldsymbol{b} \cdot(\boldsymbol{\nabla} \times \boldsymbol{b})\rangle$, i.e. the linkage between electric current lines, are used to this end (where $\boldsymbol{b}$ represents magnetic fluctuations). These quantities are useful in theoretical studies of dynamo saturation also and we use them below. Our work needs a clear distinction between such concepts as $\alpha$ effect and the corresponding helicity, and between helicities of total magnetic field, large-scale magnetic field and small-scale magnetic field; these distinctions can be neglected to some extent in other areas of dynamo theory.

\section{Magnetic and current helicity data obtained at the Huairou Solar Observing Station}

The averaged value of the small-scale magnetic helicity, i.e. $\langle\boldsymbol{a} \cdot \boldsymbol{b}\rangle$, evidently would be a convenient quantity to confront with a dynamo saturation scenario based on a magnetic helicity conservation argument. In practice however, the vector potential $\boldsymbol{a}$, being a non-gauge invariant quantity is inconvenient observationally, and it is the current helicity $\langle\boldsymbol{b} \cdot(\nabla \times \boldsymbol{b})\rangle=$ $\left\langle b_{x}(\nabla \times \boldsymbol{b})_{x}\right\rangle+\left\langle b_{y}(\nabla \times \boldsymbol{b})_{y}\right\rangle+\left\langle b_{z}(\nabla \times \boldsymbol{b})_{z}\right\rangle$ which can be extracted from the observations (here $x, y, z$ are local cartesian coordinates connected with a point on the solar surface and the $z$-axis is normal to the surface). The observations are restricted to active regions on the solar surface and we obtain information concerning the surface magnetic field and helicity only. Monitoring of solar active regions while they are passing near to the central meridian of the solar disc enables observers to determine the full magnetic field vector. The observed magnetic field is subjected to further analysis to obtain the value $\boldsymbol{\nabla} \times \boldsymbol{b}$. Because it is calculated from the surface magnetic field distribution, the only electric current component that can be calculated is $(\boldsymbol{\nabla} \times \boldsymbol{b})_{z}$. As a consequence of these restrictions, the observable quantity is

$H_{\mathrm{c}}=\left\langle b_{z}(\boldsymbol{\nabla} \times \boldsymbol{b})_{z}\right\rangle$,

which can be rewritten in the form

$H_{\mathrm{c}}=\left\langle h_{z}(\boldsymbol{\nabla} \times \boldsymbol{h})_{z}\right\rangle-B_{z}(\boldsymbol{\nabla} \times \boldsymbol{B})_{z}$,

where $\boldsymbol{h}=\boldsymbol{B}+\boldsymbol{b}$ is the total magnetic field, $\boldsymbol{B}$ is the mean magnetic field and $\boldsymbol{b}$ are the magnetic fluctuations.

Because the surface magnetic field is almost force-free, it is also useful to consider the magnetic field twist $a_{\mathrm{ff}}$ (Woltjer 1958) which is defined as the proportionality coefficient between magnetic field and electric current $\left(\boldsymbol{j}=a_{\mathrm{ff}} \boldsymbol{b}\right)$. The observational restrictions discussed above imply that the observational equivalent of twist is

$a_{\mathrm{ff}}=j_{z} / b_{z}$.

The observational data used in our analysis were obtained at the Huairou Solar Observing station of the National Astronomical Observatories of China. The magnetographic instrument based on the FeI $5324 \AA$ spectral line determines the magnetic field values at the photospheric level. The data are obtained from a CCD camera with $512 \times 512$ pixels over the whole magnetogram, whose entire size is comparable with the size of an active region, as well as with the depth of the solar convective zone (about $2 \times 10^{8} \mathrm{~m}$ ). However, because of the observational technique, the line-of-sight field component $b_{z}$ can be determined with a much higher precision than the transverse components $\left(b_{x}\right.$ and $\left.b_{y}\right)$. There are a number of other observational difficulties such as in resolving the so-called " $180^{\circ}$ ambiguity" in the direction of the transverse field etc. The observational technique is described in detail by Wang et al. (1996), see also Abramenko et al. (1996).

An observational programme to reveal the values of the twist and the current helicity density over the solar surface requires a systematic approach, both to the monitoring of magnetic fields in active regions and to the data reduction, in order to reduce the impact of noise. This work has been carried out by a number of research groups (e.g. Seehafer 1990; 
Table 1. The first column gives the central latitude $\Theta=90^{\circ}-\theta$ of the data bin, with the averaging interval in brackets, the twist $\left\langle a_{\mathrm{ff}}\right\rangle$ is measured in units of $10^{-8} \mathrm{~m}^{-1}$, the current helicity $\left\langle H_{\mathrm{c}}\right\rangle$ in units of $10^{-3} \mathrm{G}^{2} \mathrm{~m}^{-1}$, and $N$ is the number of active regions involved in the analysis. The errors correspond to the $95 \%$ confidence level, i.e. about two standard deviations.

\begin{tabular}{crrr}
\hline \hline$\Theta$ & $\left\langle a_{\mathrm{ff}}\right\rangle$ & $\left\langle H_{\mathrm{c}}\right\rangle$ & $N$ \\
\hline $28(24-32)$ & $-0.4 \pm 1.2$ & $-1.6 \pm 1.7$ & 18 \\
$20(16-24)$ & $-0.9 \pm 0.8$ & $-0.9 \pm 0.4$ & 51 \\
$14(12-16)$ & $-1.7 \pm 1.3$ & $-0.6 \pm 0.4$ & 34 \\
$10(8-12)$ & $-2.2 \pm 0.6$ & $-0.4 \pm 0.2$ & 49 \\
$4(0-8)$ & $-1.9 \pm 0.8$ & $-0.6 \pm 0.2$ & 44 \\
$-4(-8-0)$ & $0.3 \pm 0.7$ & $0.7 \pm 0.5$ & 31 \\
$-10(-12--8)$ & $1.2 \pm 0.7$ & $0.7 \pm 0.4$ & 59 \\
$-14(-16--12)$ & $0.9 \pm 0.7$ & $0.9 \pm 0.7$ & 46 \\
$-20(-24--16)$ & $1.0 \pm 0.8$ & $0.4 \pm 0.2$ & 68 \\
$-28(-32--24)$ & $1.6 \pm 1.7$ & $0.5 \pm 0.9$ & 14 \\
\hline
\end{tabular}

Table 2. The data of Table 1 binned by hemisphere and year of observation.

\begin{tabular}{cccr}
\hline \hline$T$ & $\left\langle a_{\mathrm{ff}}\right\rangle$ & $\left\langle H_{\mathrm{c}}\right\rangle$ & $N$ \\
\hline \multicolumn{4}{c}{ North } \\
\hline $1988-89$ & $-1.1 \pm 0.8$ & $-1.0 \pm 0.5$ & 50 \\
$1990-91$ & $-1.0 \pm 0.7$ & $-1.0 \pm 0.5$ & 61 \\
$1992-93$ & $-2.1 \pm 0.7$ & $-0.7 \pm 0.3$ & 45 \\
$1994-95$ & $-2.6 \pm 0.9$ & $-0.3 \pm 0.1$ & 34 \\
$1996-97$ & $-1.2 \pm 1.0$ & $-0.2 \pm 0.2$ & 9 \\
\hline \multicolumn{4}{c}{ South } \\
\hline $1988-89$ & $1.0 \pm 1.2$ & $0.2 \pm 0.3$ & 38 \\
$1990-91$ & $0.9 \pm 0.7$ & $0.8 \pm 0.6$ & 65 \\
$1992-93$ & $1.2 \pm 0.5$ & $0.9 \pm 0.3$ & 77 \\
$1994-95$ & $0.7 \pm 0.9$ & $0.1 \pm 0.1$ & 35 \\
$1996-97$ & $0.3 \pm 2.0$ & $0.2 \pm 0.3$ & 8 \\
\hline \multicolumn{4}{c}{}
\end{tabular}

Pevtsov et al. 1994; Rust \& Kumar 1996; Abramenko et al. 1997; Bao \& Zhang 1998; Kuzanyan et al. 2000). While this work is still in progress, the largest systematic data-set of active regions presently available consists of 422 active regions over the 10 years 1988-1996 (Bao \& Zhang 1998). We use averages and confidence intervals calculated from these data (Table 1). Note that both the averaged quantities of twist and current helicity density are positive/negative over Southern/Northern solar hemispheres respectively, and thus obey the so-called hemispheric rule (Table 2).

Observations at Huairou Observing Station also give dopplergrams of velocity fields $\boldsymbol{v}$ over active regions from the FeI $5324 \AA$ spectral line in the photosphere and from the $H_{\beta}$ line in the chromosphere. The processing of these data could provide values of $v_{z} b_{z}$ (which is related to the so-called crosshelicity) in the foreseeable future. However, at present this quantity is not available for statistical studies.

\section{The dynamo model}

We describe the solar dynamo by means of the mean-field equation (e.g. Moffatt 1978; Parker 1979; Krause \& Rädler 1980; Zeldovich et al. 1983) which in general form is

$\frac{\partial \boldsymbol{B}}{\partial t}=\boldsymbol{\nabla} \times\left(\boldsymbol{V} \times \boldsymbol{B}+\mathcal{E}-\eta_{0} \boldsymbol{\nabla} \times \boldsymbol{B}\right)$,

where $\boldsymbol{V}$ is a mean velocity (e.g. the differential rotation), $\eta_{0}$ is the magnetic diffusion due to the electrical conductivity of the fluid, $\mathcal{E}=\langle\boldsymbol{u} \times \boldsymbol{b}\rangle$ is the mean electromotive force, $\boldsymbol{u}$ and $\boldsymbol{b}$ are fluctuations of the velocity and magnetic field respectively, and angular brackets denote averaging over an ensemble of fluctuations. The electromotive force $\mathcal{E}$ can be separated into several contributions, which include the $\alpha$-effect, turbulent magnetic diffusivity $\eta$ and other terms such as the magnetic turbulent diamagnetic effect. For now, we restrict ourself to the two first terms, and consider $\alpha$ and $\eta$ to be isotropic quantities. We take the turbulent diffusivity as a prescribed quantity and take into account the nonlinearity of the $\alpha$-effect only, i.e. we use the parameterization

$\boldsymbol{E}=\alpha(\boldsymbol{B}) \boldsymbol{B}-\eta \boldsymbol{\nabla} \times \boldsymbol{B}$,

where $\alpha$ depends in principle on the entire evolution of the magnetic field, rather on its value in a given instant. This dependence is described by an evolution equation (such as Eqs. (8) and (13) below).

Using spherical coordinates $r, \theta, \phi$, we represent an axisymmetric mean magnetic field as $\boldsymbol{B}=B_{\phi} \boldsymbol{e}_{\phi}+\boldsymbol{\nabla} \times\left(A \boldsymbol{e}_{\phi}\right)$. Following Parker (1955) we consider dynamo action in a thin convective shell, average $A$ and $B_{\phi}$ over the depth of the convective shell and consider these quantities as functions of colatitude $\theta$ only. Then we neglect the curvature of the convective shell and replace it by a flat slab to get the following equations (we drop the suffix on $B_{\phi}$ for the sake of brevity)

$$
\begin{aligned}
& \frac{\partial B}{\partial t}=g D \sin \theta \frac{\partial A}{\partial \theta}+\frac{\partial^{2} B}{\partial \theta^{2}}-\mu^{2} B, \\
& \frac{\partial A}{\partial t}=\alpha B+\frac{\partial^{2} A}{\partial \theta^{2}}-\mu^{2} A
\end{aligned}
$$

(see Appendix A). Here we measure lengths in units of the solar radius and time in units of a diffusion time based on the solar radius and turbulent magnetic diffusivity. The terms $-\mu^{2} B$ and $-\mu^{2} A$ represent the role of turbulent diffusive losses in the radial direction - the value $\mu=3$ corresponds to a convective zone with a thickness of about $1 / 3$ of the solar radius. $g=\partial \Omega / \partial r$ is the radial shear of differential rotation. We neglect any latitudinal dependence of the rotation curve as well as the link between poloidal and toroidal magnetic field via the $\alpha$-effect, and so consider a simple $\alpha \omega$-dynamo. $\alpha$ and $g$ are normalized with respect to their maximal values and incorporated into the dimensionless dynamo number $D$, which gives the intensity of the dynamo action (see Sokoloff et al. 1995 for mathematical details of the derivation of Eqs. (5) and (6) from Eqs. (3) and (4)).

These equations are obviously oversimplified. Starting from the fundamental paper of Parker (1955) they can be used however to reproduce basic qualitative features of solar and 
stellar activity and appear to be viable for this purpose. Taking into account the nature of the approach, we use the simplest profiles of dynamo generators compatible with symmetry requirements, i.e. $\alpha(\theta)=\cos \theta$ and $g=1$.

The points $\theta=0$ and $\theta=180^{\circ}$ correspond to North and South poles respectively. We take here zero boundary conditions for $A$ and $B$. (Because we neglect the convective shell curvature, these boundary conditions necessarily are approximate.) In principle, we could use a slightly more elaborate version of Eqs. (5) and (6) which take into account some curvature effects, so the diffusion term becomes formally singular at the poles and a more realistic finiteness condition can be exploited (see Galitski \& Sokoloff 1999). Magnetic helicity data are available for middle latitudes and the equatorial region $\left(-30^{\circ}<\Theta<30^{\circ}\right.$ where the latitude $\Theta=90^{\circ}-\theta$ and $\Theta=0$ corresponds to the equator) only and so here we are not very interested in details of dynamo wave behaviour near to the poles. We keep a factor $\sin \theta$ in Eq. (5) which reflects the fact that the length of the parallels $\theta=$ const vanishes at the poles (Kuzanyan \& Sokoloff 1995). Neglecting this term results in an unphysical coupling between the dynamo wave behaviour near to the pole and near to the equator.

We are interested in dynamo waves propagating from middle solar latitudes towards the equator. This corresponds to negative dynamo numbers provided $\alpha$ is chosen to be positive in the Northern hemisphere and $g$ is positive near to the solar equator. According to various models, the ranges of $|D| \approx 10^{3}-10^{6}$ can be considered as realistic for the solar case.

We nondimensionalize the dynamo equations by measuring length in units of the solar radius $R$, time in units of the turbulent magnetic diffusion time $R^{2} / \eta$, and the differential rotation $\delta \Omega$ in units of the maximal value of $\Omega$. $\alpha$ is measured in units of the maximum value of the hydrodynamic part of the $\alpha$-effect.

It is convenient to present the dynamo number as $D=$ $R_{\alpha} R_{\omega}$, where $R_{\alpha}=\alpha R / \eta \sim 1-200$ and $R_{\omega}=\delta \Omega R^{2} / \eta \sim$ $(1-4) \times 10^{3}$ (where typical values of parameters have been used for these estimates) represent the contributions of the $\alpha$-effect and differential rotation, respectively. We use the equipartition magnetic field $B_{*}=u \sqrt{4 \pi \rho}$ as the unit of magnetic field. The vector potential of the poloidal field $A$ is measured in units of $R_{\alpha} R B_{*}$, the density $\rho$ normalized to its value at the bottom of the convective zone, and the basic scale of the turbulent motions $l$ and turbulent velocity $u$ at the scale $l$ are measured in units of their maximum values through the convective region. The magnetic Reynolds number $\mathrm{Rm}=l u / \eta_{0}$ is defined using these maximal values. $\eta=l u / 3$ is an estimate for the turbulent diffusivity.

We stress that all physical ingredients of the model vary more-or-less strongly with the depth $h_{*}$ below the solar surface and we have to use some average quantities in the Parker dynamo equations. We use mainly estimates of governing parameters taken from models of the solar convective zone (see, e.g., Spruit 1974; Baker \& Temesvary 1966; more modern treatments make little difference to these estimates). In particular, at depth $h_{*} \sim 2 \times 10^{10} \mathrm{~cm}, \mathrm{Rm} \sim 2 \times 10^{9}, u \sim 2 \times 10^{3} \mathrm{~cm} \mathrm{~s}^{-1}$, $l \sim 8 \times 10^{9} \mathrm{~cm}, \rho \sim 2 \times 10^{-1} \mathrm{~g} \mathrm{~cm}^{-3}, \eta \sim 5.3 \times 10^{12} \mathrm{~cm}^{2} \mathrm{~s}^{-1}$. The density stratification scale is estimated here as $\Lambda_{\rho}=$ $\rho /|\nabla \rho| \sim 6.5 \times 10^{9} \mathrm{~cm}$ and the equipartition mean magnetic field $B_{*}=3000 \mathrm{G}$. In the upper part of the convective zone, say at depth $h_{*} \sim 2 \times 10^{7} \mathrm{~cm}$, these parameters are $\mathrm{Rm} \sim 10^{5}$, $u \sim 9.4 \times 10^{4} \mathrm{~cm} \mathrm{~s}^{-1}, l \sim 2.6 \times 10^{7} \mathrm{~cm}, \rho \sim 4.5 \times 10^{-7} \mathrm{~g} \mathrm{~cm}^{-3}$, $\eta \sim 0.8 \times 10^{12} \mathrm{~cm}^{2} \mathrm{~s}^{-1}$ and $\Lambda_{\rho} \sim 3.6 \times 10^{7} \mathrm{~cm}$; the equipartition mean magnetic field is $B_{*}=220 \mathrm{G}$ here. This estimate for the equipartition magnetic field at the base of the convection zone $\left(B_{*}=3000 \mathrm{G}\right)$ is roughly consistent with the magnetic field strength in sunspots (about $1 \mathrm{kG}$ ). However obviously it should be distinguished from the mean magnetic field at the solar surface; a deeper discussion of this distinction is outside of the scope of the paper. For the Parker migratory dynamo, the toroidal magnetic field usually dominates and below we ignore the poloidal magnetic field when calculating the magnetic energy.

\section{The nonlinearities}

A key idea of the dynamo saturation scenario exploited below is a splitting of the total $\alpha$ effect into the hydrodynamic $\left(\alpha^{v}\right)$ and magnetic $\left(\alpha^{m}\right)$ parts

$\alpha(r, \theta)=\alpha^{v}+\alpha^{m}$,

as first suggested by Frisch et al. (1975). We need to parameterize both contributions, $\alpha^{v}$ and $\alpha^{m}$, in terms of the magnetic field components and helicities. Two types of effect should be taken into account. First of all, the link between $\alpha$-effect and the relevant helicities can be modified by the dynamo-generated magnetic field. Correspondingly, we introduce quenching functions $\phi_{v}\left(\alpha^{v}=\chi^{v} \phi_{v}\right.$ with $\left.\chi^{v}=-(\tau / 3)\langle\boldsymbol{u} \cdot(\nabla \times \boldsymbol{u})\rangle\right)$ and $\phi_{m}\left(\alpha^{m}=\chi^{c} \phi_{m}\right.$, and $\chi^{c}$ is defined below) to obtain $\alpha=\chi^{v} \phi_{v}+\chi^{c} \phi_{m}$, where $\tau$ is the correlation time of the turbulent velocity field.

The second problem to be addressed is that magnetic helicity is not a very convenient quantity because it involves a gauge-noninvariant quantity, i.e. the vector potential. We connect magnetic helicity with the current helicity $\langle\boldsymbol{b} \cdot(\boldsymbol{\nabla} \times \boldsymbol{b})\rangle$, by using the approximation of locally homogeneous turbulent convection (see Kleeorin \& Rogachevskii 1999). Then we need to obtain a quantity of suitable dimension, and introduce the density $\rho$ to obtain the correctly dimensioned $\chi^{c} \equiv$ $(\tau / 12 \pi \rho)\langle\boldsymbol{b} \cdot(\boldsymbol{\nabla} \times \boldsymbol{b})\rangle$. Thus,

$\alpha(r, \theta)=\chi^{v} \phi_{v}+\chi^{c} \phi_{m}$.

The issue of the large-scale and small-scale current helicities was discussed by Dikpati \& Gilman (2001) and by Brandenburg et al. (2002).

The quenching functions $\phi_{v}$ and $\phi_{m}$ in Eq. (8) are given by

$\phi_{v}(B)=(1 / 7)\left[4 \phi_{m}(B)+3 L(B)\right]$,

$\phi_{m}(B)=\frac{3}{8 B^{2}}[1-\arctan (\sqrt{8} B) / \sqrt{8} B]$

(see Rogachevskii \& Kleeorin 2000, 2001), where $L(B)=1-$ $16 B^{2}+128 B^{4} \ln \left(1+1 /\left(8 B^{2}\right)\right)$. Thus $\phi_{v}=1 /\left(4 B^{2}\right)$ and $\phi_{m}=$ $3 /\left(8 B^{2}\right)$ for $B \gg 1 / 3$; and $\phi_{v}=1-(48 / 5) B^{2}$ and $\phi_{m}=1-$ $(24 / 5) B^{2}$ for $B \ll 1 / 3$. Here $\chi^{v}$ and $\chi^{c}$ are measured in units of the maximal value of the $\alpha$-effect.

The function $\phi_{v}$ describes conventional quenching of the $\alpha$ effect. A simple form of such a quenching, $\phi_{v}=1 /\left(1+B^{2}\right)$, was 
introduced long ago (see, e.g. Iroshnikov 1970). This form is quite close to the more sophisticated form presented in Eq. (9). The magnetic part $\alpha^{m}$ includes two types of nonlinearity: the algebraic quenching described by the function $\phi_{m}$ (see e.g. Field et al. 1999; Rogachevskii \& Kleeorin 2000, 2001) and the dynamic nonlinearity which is determined by Eq. (13).

The quenching of the $\alpha$-effect is caused by the direct and indirect modification of the electromotive force by the mean magnetic field. The indirect modification of the electromotive force is caused by the effect of the mean magnetic field on the velocity fluctuations and on the magnetic fluctuations, while the direct modification is due to the effect of the mean magnetic field on the cross-helicity (see, e.g., Rogachevskii \& Kleeorin 2000, 2001).

We can calculate also the cross-helicity $\langle\boldsymbol{u} \cdot \boldsymbol{b}\rangle$ which may in the future be compared with observational data

$\langle\boldsymbol{u} \cdot \boldsymbol{b}\rangle=(\eta / 2)\left[3 \Lambda_{u}^{-1} B_{r}+\phi_{\mathrm{ch}}(B)(\boldsymbol{B} \cdot \boldsymbol{\nabla}) B^{2}\right]$,

where $\Lambda_{u}^{-1}=\left|\boldsymbol{\nabla}\left\langle\boldsymbol{u}^{2}\right\rangle\right| /\left\langle\boldsymbol{u}^{2}\right\rangle, \quad \phi_{\mathrm{ch}}(B)=\left(2 / 35 B^{2}\right)[(15+$ $\left.\left.224 B^{2}\right) \phi_{m}(2 B)+6 L(2 B)-21\right]$, and $\phi_{\mathrm{ch}}(B)=-128 / 5$ for $B \ll$ $1 / 3$, and $\phi_{\mathrm{ch}}(B)=-3 \pi /\left(20 \sqrt{2} B^{3}\right)$ for $B \gg 1 / 3$. When deriving Eq. (11) we used Eqs. (A14)-(A17) and (A21) of Rogachevskii \& Kleeorin (2001).

Now we need to average Eq. (8) over the depth of the convective zone. The first term in the averaged equation seems to be determined by the values taken at some sort of mean position in the convective zone, while the situation concerning the second term is much less clear, because the density used to calculate $\chi^{c}$ decreases strongly with radius. The clarification of this problem is obviously beyond the Parker approximation; however to address this problem as far as it possible here we introduce a phenomenological parameter $\sigma$ by

$\alpha(\theta)=\chi^{v} \phi_{v}+\sigma \chi^{c} \phi_{m}$,

where the helicities and quenching functions are associated with some sort of mean position in the convective zone. We emphasize that below we consider $\sigma$ as a free parameter in the context of the averaging process used to derive the Parker equations; probably we can only safely assert that $\sigma \gtrsim 1$.

For the sake of brevity of notation, we keep in Eq. (12) the same notation as in Eqs. (7) and (8). We stress that now we consider a parameterization for helicities which depends on colatitude $\theta$ only. Of course, this is not more than a phenomenological description, to be improved in more detailed models of the nonlinear solar dynamo.

The function $\chi^{c}(\boldsymbol{B})$ is determined by a dynamical equation which follows from the conservation law for magnetic helicity (see Kleeorin \& Rogachevskii 1999). A general dimensional form of this equation reads

$\frac{\partial \chi^{c}}{\partial t}+\frac{\chi^{c}}{T}=-\frac{1}{9 \pi \eta \rho_{*}}(\boldsymbol{E} \cdot \boldsymbol{B}+\boldsymbol{\nabla} \cdot \boldsymbol{\Phi})+\kappa \Delta \chi^{c}$.

Here $\boldsymbol{\Phi}=C \chi^{v} \phi_{v} \boldsymbol{B}^{2} l^{2} \boldsymbol{e}_{\mathrm{r}} / \Lambda_{\rho}$ is a nonadvective flux of the magnetic helicity (here $\boldsymbol{e}_{\mathrm{r}}$ is the unit vector in radial direction), $-\kappa \nabla \chi^{c}$ is the diffusive flux of the magnetic helicity (see Kleeorin \& Rogachevskii 1999; Kleeorin et al. 2000, 2002, 2003), and $T=l^{2} / \eta_{0}$ is the relaxation time of magnetic helicity.
Magnetic helicity transport through the boundary of a dynamo region is reported by Chae (2001) to be observable at the solar surface. The flux of magnetic helicity for the sun has been estimated by Berger \& Ruzmaikin (2000). The evolution of large-scale magnetic helicity as well as the associated magnetic helicity fluxes have been calculated from twodimensional models by Brandenburg et al. (2002).

We also take into account that for an axisymmetric problem the term which determines the advective flux of the magnetic helicity, $\boldsymbol{\nabla} \cdot\left(\boldsymbol{V} \chi^{c}\right)$, vanishes $\left(\boldsymbol{V}=\boldsymbol{e}_{\phi} \Omega r \sin \theta\right.$ is the differential rotation). The parameter $C$ is a numerical coefficient and $\kappa$ is of order $\eta$. In principle, these parameters can be calculated given some model of convection, however we here take into account our real level of knowledge and keep them as free parameters. Note that in estimating the helicity flux $\boldsymbol{\Phi}$ we have to include density gradients in the radial direction which are neglected in other parts of the analysis.

The physical meaning of Eq. (13) is that the total magnetic helicity is a conserved quantity and if the large-scale magnetic helicity grows with magnetic field, the evolution of the small-scale helicity should somehow compensate this growth. Compensation mechanisms include dissipation and various kinds of transport.

The dynamical Eq. (13) for the function $\chi^{c}(\boldsymbol{B})$ in nondimensional form in the context of the Parker migratory dynamo reads

$$
\begin{aligned}
\frac{\partial \chi^{c}}{\partial t}+ & \left(T^{-1}+\kappa \mu^{2}\right) \chi^{c}=\left(\frac{2 R}{l}\right)^{2}\left(\frac{\partial A}{\partial \theta} \frac{\partial B}{\partial \theta}-B \frac{\partial^{2} A}{\partial \theta^{2}}\right. \\
& \left.-\alpha B^{2}+2 \mu^{2} A B+C B^{2} \phi_{v} \chi^{v}(\theta)\right)+\kappa \frac{\partial^{2} \chi^{c}}{\partial \theta^{2}}
\end{aligned}
$$

(see Appendix A), where we have averaged Eq. (13) over the depth of the convective zone, so that the averaged value of $T^{-1}$ is

$T^{-1}=H^{-1} \int T^{-1}(r) \mathrm{dr} \sim \frac{\Lambda_{l} R^{2} \eta_{0}}{H l^{2} \eta} \approx 0.2-0.5$,

$\Lambda_{l}$ is the characteristic scale of the variations $l, T(r)=$ $\left(\eta / R^{2}\right)\left(l^{2} / \eta_{0}\right)$ is the nondimensional relaxation time of magnetic helicity, and the quantities $\Lambda_{l}, \eta_{0}, l$ in Eq. (15) are associated with the upper part of the convective zone. The parameters $C$ and $\kappa$ can be chosen as $C \sim(0.1-1) \times 10^{-1}$ and $\kappa=0.1-1$. The factor $10^{-1}$ in the coefficient $C$ arises from the parameter $\left(l^{2} / \Lambda_{\rho}\right) / R \sim 0.1$ (see Appendix A).

\section{Results}

We simulated numerically the model of the nonlinear solar dynamo based on the Parker approximation and conservation of magnetic helicity arguments, as presented in previous sections. We found that the model gives a stable nonlinear wave-type solution similar to the solar cycle phenomenology from the generation threshold of the nonlinear system $\left(D_{\text {nonl,crit }} \approx-940\right.$ with $\sigma=\kappa=1, C=0.1)$ up to $D=-10^{4}$. It is quite interesting that a slightly stronger generation is needed to get stable nonlinear oscillations with a nonvanishing amplitude than to excite the linear dynamo, where $D_{\text {crit }} \approx-910$. The temporal behaviour of 
Table 3. Parameters of activity cycles: $B_{\max }$ is the value of the dimensionless amplitude of the toroidal magnetic field in units of the equipartition field (estimated as $3000 \mathrm{G}$ at the bottom of the convective zone), $T_{\mathrm{c}}$ is the dimensionless cycle length, SP and DP denote singly and doubly periodic solutions respectively; "runs away" means that no stable finite amplitude solution was found, although in some cases there is a long pseudo-stable initial phase. The other governing parameters are $\sigma=1, T=3,(2 R / l)^{2}=300$. For the DP solutions, the amplitude of the stronger cycles and the shorter period are given.

\begin{tabular}{llrlll}
\hline \hline$C$ & $\kappa$ & $D$ & $B_{\max }$ & $T_{\mathrm{c}}$ & \\
\hline 0.1 & 1 & $-10^{3}$ & 0.12 & 0.37 & $\mathrm{SP}$ \\
0.1 & 1 & $-10^{4}$ & 5.28 & 0.25 & $\mathrm{DP}$ \\
0.1 & 1 & $-2 \times 10^{4}$ & - & - & runs away \\
0.1 & 0.1 & $-10^{3}$ & 0.09 & 0.345 & $\mathrm{SP}$ \\
0.1 & 0.1 & $-10^{4}$ & - & - & runs away \\
0.1 & 3 & $-10^{3}$ & 0.13 & 0.355 & $\mathrm{SP}$ \\
0.1 & 3 & $-3 \times 10^{3}$ & 1.47 & 0.46 & $\mathrm{SP}$ \\
0.1 & 3 & $-10^{4}$ & - & - & runs away \\
0.01 & 1 & $-10^{3}$ & 0.11 & 0.35 & $\mathrm{SP}$ \\
0.01 & 1 & $-3 \times 10^{3}$ & 1.35 & 0.37 & $\mathrm{SP}$ \\
0.01 & 1 & $-10^{4}$ & 4.50 & 0.30 & $\mathrm{DP}$ \\
0.01 & 1 & $-2 \times 10^{4}$ & - & - & runs away \\
0 & 1 & $-10^{4}$ & 4.25 & 0.29 & weakly DP \\
0 & 1 & $-2 \times 10^{4}$ & - & - & runs away \\
\hline \multicolumn{7}{c}{} & & & &
\end{tabular}

nonlinear dynamo waves is quite similar to that with the simple algebraic $\alpha$-quenching, and we give here, instead of quite standard plots, details of the cycle parameters in Table 3. A feature of the activity cycles illustrated by this Table is a transition from singly to doubly periodic solutions with growth of dynamo intensity.

In the present paper we have concentrated on the dynamics of the current helicity (which is proportional to the function $\chi^{c}$ ) and its comparison with the observations. At the present state of observations we can compare latitudinal distributions of $\chi^{c}$ averaged over the activity cycle or the temporal behaviour of $\chi^{c}$ averaged over a hemisphere. Such comparisons are presented in Figs. $1-3$ for $D=-10^{3}, \sigma=1, T=3,(2 R / l)^{2}=300$. In Figs. 1 and 3 the parameter $\kappa=0.1$. We restrict ourselves to discussing dynamo models with singly periodic behaviour (although there are hints of a double periodicity in the sunspot record). Two types of behaviour are demonstrated. Provided that $C$ is negative (magnetic helicity inflow, see Fig. 2b) or small and positive $(C<0.1$, moderate magnetic helicity outflow, see Fig. 2a) the value of $\chi^{c}$ is always negative in the Northern hemisphere, in accordance with naive theoretical expectations as well as the available observations. If $C$ is large and positive (strong magnetic helicity outflow), we obtain a cycle during which $\chi^{c}$ changes sign. Both types of behaviour are shown in Fig. 1, for $C=0.01$ (solid curve) and $C=0.1$ (broken). Comparing observational and theoretical results we have to fit the numerical data for $\chi^{c}$ with its observational equivalent $H_{\mathrm{c}}$. We normalize the data to make them equal at $\Theta=28^{\circ}\left(\left\langle\chi^{c}\right\rangle_{\Theta=28^{\circ}}=23.5\left\langle H_{\mathrm{c}}\right\rangle\right)$. Because of this fitting procedure, the agreement between the observational and numerical data is much better in the Northern hemisphere than in the Southern.

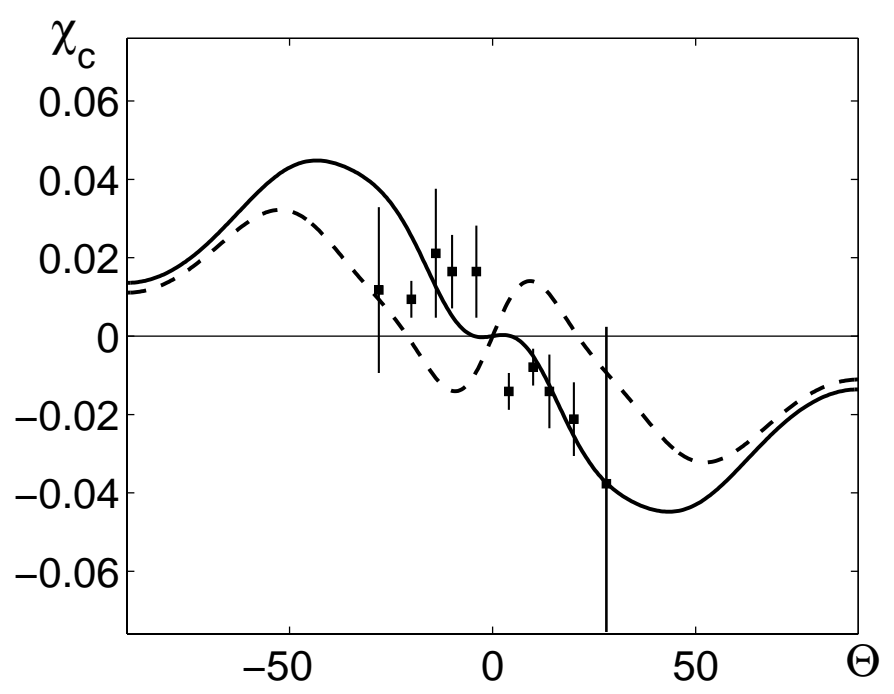

Fig. 1. The latitude dependence of the time-averaged function $\left\langle\chi^{c}\right\rangle$ for various parameter sets (see description in the text). The observed values of the time-averaged $\left\langle H_{\mathrm{c}}\right\rangle$ are shown by filled squares, the error-bars are shown by vertical lines. A fitting factor of 23.5 , (i.e., $\left.\left\langle\chi^{c}\right\rangle_{\Theta=28^{\circ}}=23.5\left\langle H_{c}\right\rangle\right)$ has been used - see text.

We illustrate the importance of the fitting procedure for various parameter sets in Fig. 2 where we give the results for $C=0.01$ in panel a and for $C=-0.1$ in panel $\mathrm{b}$. The results for $\kappa=0.1$ are shown by broken curves and those for $\kappa=1$ by solid. Both cases represent a model which does not exhibit helicity reversals. We appreciate that the agreement between numerical and observational data in the Northern hemisphere can be partially attributed to the fitting procedure; however the agreement obtained looks quite impressive for the primitive models considered. A disagreement between the model and observations at $\Theta=4^{\circ}$ can be explained, for example, as a result of a non-perfect North-South symmetry in the observed cycle. We conclude from Fig. 2 that the minimal value of $\left\langle\chi^{c}\right\rangle$ decreases with $\kappa$; experience from numerical simulations show that it decreases with $\sigma$ as well. Note however that the contribution of $\chi^{c}$ to the magnetic part of the $\alpha$-effect is determined by $\sigma\left\langle\chi^{c}\right\rangle$ and this value is more or less stable.

Moving to the comparison of the temporal helicity evolution presented in Fig. 3, we note that the fitting procedures now have to be more complicated. The first two measured points (1988-89) and (1990-91) with approximately equal values of the latitude-averaged $\left\langle H_{\mathrm{c}}\right\rangle$ are chosen to be located symmetrically about the minimum of the function $\left\langle\chi^{c}\right\rangle$ for $\Theta>0$. The distance between the observational points is $(2 / 11)\left(T_{\mathrm{c}} / 2\right)$, where $T_{\mathrm{c}}$ is the period of oscillations of the toroidal magnetic field. The fitting factor is 44.3 , determined by the condition $\left\langle\chi^{c}\right\rangle_{t=3.527 t_{\mathrm{d}}}=44.3\left\langle H_{\mathrm{c}}\right\rangle_{t=1988}$, where $t_{\mathrm{d}}$ is the turbulent diffusion time at the bottom of the convective zone.

The point is that we can base the fitting procedure on a comparison between the simulated value of the toroidal magnetic field and some tracer of cyclic solar activity, say, the averaged group sunspot number $R_{\mathrm{g}}$ (Hoyt et al. 1994) ${ }^{1}$. This fitting is also

1 The data for $R_{\mathrm{g}}$ were taken from the URL ftp.ngdc.noaa.gov/STP/ 

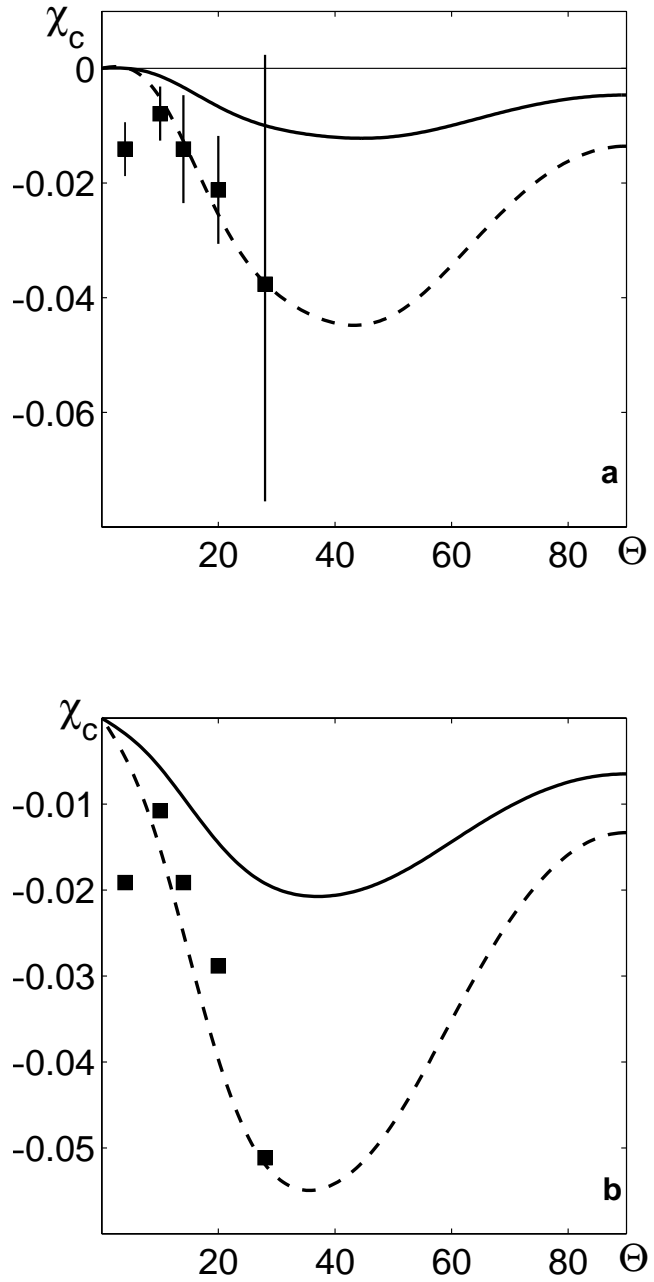

Fig. 2. The latitude dependence of the time-averaged function $\left\langle\chi^{c}\right\rangle$ for various parameter sets (see description in the text). The observed values of the time-averaged $\left\langle H_{\mathrm{c}}\right\rangle$ are shown by filled squares, the errorbars are shown by vertical lines. We have used a fitting factor in a) of 23.5, (i.e., $\left.\left\langle\chi^{c}\right\rangle_{\Theta=28^{\circ}}=23.5\left\langle H_{\mathrm{c}}\right\rangle\right)$ and in panel b) it is 32 .

shown in Fig. 3 as follows. The dashed-dotted line shows the time dependence of the latitude-averaged function $-6\left\langle B^{2}\right\rangle$ for the same parameters. The fitting factor 6 is determined by the condition $6\left\langle B^{2}\right\rangle_{t=3.527 t_{d}}=0.07$. The values $-R_{\mathrm{g}} / 2160$ are shown in Fig. 3 by the filled diamonds. The fitting factor $1 / 2160$ is determined by the condition: $R_{\mathrm{g}}(t=1988) / 2160=0.07$. (Our slightly awkward looking choice of scale for some of the quantities plotted in Fig. 3 arises because we choose to fit our model in the Northern hemisphere where $\left\langle\chi^{c}\right\rangle$ and $\left\langle H_{\mathrm{c}}\right\rangle$ are negative.)

It can be seen from Fig. 3 that the maxima of the magnetic energy $\left\langle B^{2}\right\rangle$ nearly coincide with the minima of the function $\chi^{c}$. A similar behaviour was observed also for the quantity $R_{\mathrm{g}}$ (a tracer of cyclic solar activity) and $H_{\mathrm{c}}$ (the tracer of the current helicity), cf. Bao \& Zhang (1998).

\section{Discussion and conclusions}

We have presented above a simple model of the nonlinear solar cycle with a dynamo saturation mechanism based on magnetic helicity conservation. The model is obviously oversimplified, however it reproduces some features of real stellar cycles

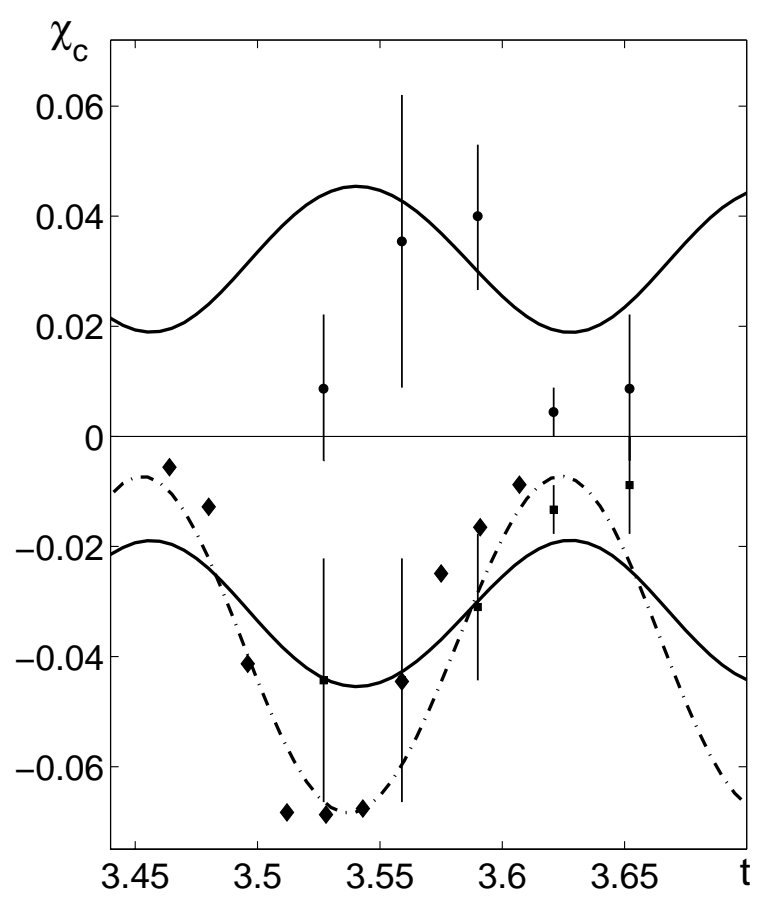

Fig. 3. The time dependence of the latitude-averaged function $\left\langle\chi^{c}\right\rangle$ for $D=-10^{3}, \sigma=1, C=0.01$ and $\kappa=0.1$. The observed values of the latitude-averaged function $\left\langle H_{\mathrm{c}}\right\rangle$ are shown by filled squares (for $\Theta>0$ - Northern hemisphere, lower panel) and filled circles (for $\Theta<0$ Southern hemisphere, upper panel), the error-bars for $\left\langle H_{\mathrm{c}}\right\rangle$ are shown by vertical lines. The dashed-dotted line indicates the time dependence of the latitude-averaged function $-6\left\langle B^{2}\right\rangle$. The filled diamonds in the lower panel give the scaled averaged group sunspot numbers, $R_{\mathrm{g}}-$ see text for further details.

as far as they are known from, say, the Wilson sample data (Baliunas et al. 1995). In particular, we obtain both singly periodic and doubly periodic cycles. As expected from qualitative arguments as well from observational data (see e.g. Bruevich et al. 2001) the transition from singly periodic to doubly periodic cycles is associated with a general trend from cycles with smaller amplitude to those with larger amplitude.

Our model of dynamo saturation gives stable oscillations for a limited region of parameter space. If the dynamo generation becomes stronger the numerical solution runs away. On one hand, we have not included in the model all kinds of nonlinear dynamo saturation; e.g. buoyancy and spot formation obviously lead to some losses of toroidal magnetic field and thus contribute to dynamo saturation. In addition, increasing the dynamo number can reduce the radial spatial scale of the toroidal magnetic field and effectively enlarge the parameter $\mu$ which determines the toroidal magnetic field dissipation. If $\mu$ is independent of $D$, we thus artificially overestimate the generation effect for larger dynamo numbers. On the other hand, experience from dynamo simulations as well as observational data suggests that cyclic behaviour is typical for moderate dynamo action only and chaotic behaviour occurs when the dynamo action is stronger. It is more than natural to expect that a parameter range with chaotic temporal behaviour will also exist in our model. 
We have concentrated our attention here on current helicity data, so a deeper comparison of the cycle parameters with observational data is beyond the scope of this paper. Also, we should keep in mind that we compare the simulated current helicity $\propto \chi^{c}$ with just one part of the surface current helicity $\left\langle b_{z}(\boldsymbol{\nabla} \times \boldsymbol{b})_{z}\right\rangle$.

The properties of current helicity simulated with our model have been compared with the available observational data. The results of this comparison look quite promising in spite of the quite limited extent of the observational data, as well as the crude nature of the model. We have been able to choose a set of governing parameters which give helicity properties comparable with the available phenomenology. From another viewpoint, the observational data are rich enough to indicate a disagreement between the available observations and the predictions of the model with other parameter sets.

The parameters which gives an agreement between simulations and observations are quite plausible. However we feel that it is too early to insist that this agreement is more than a coincidence. Really, we base our comparison on the 10 year observational data of one scientific team. We stress that an extension of the observational programme to cover several cycles as well the inclusion of data obtained by other scientific teams and from other tracers would be very important. In particular it would be valuable to include the cross-helicity data into the analysis.

Note, that although the available observations cover just the period of 10 years, these data extend over parts of two different solar cycles, namely the 22nd (1988-1995) and the 23rd (1996-1997). The available data suggest that the shape of the current helicity distribution is the same for both cycles, and further, more recent, current helicity studies (Bao et al. 2000; 2002) support this interpretation. In particular, the hemispheric rule is obeyed from cycle to cycle (e.g. Pevtsov et al. 2001).

We stress that the scenario described in the present paper does not include all possible types of nonlinear processes which can occur at the nonlinear stage of the dynamo (see, e.g., Brandenburg \& Subramanian 2000; Brandenburg \& Dobler 2001), but rather is restricted to a minimal number of processes involved in magnetic helicity conservation. In the spirit of the basic ideas about the nonlinear saturation of solar dynamos, the analysis presented here has been restricted to the evolution of $\alpha$ only, while detailed simulations (e.g. Blackman \& Brandenburg 2002; Brandenburg \& Sokoloff 2002) also demonstrate a quenching of the turbulent magnetic diffusivity. A quantitative model for a nonlinear quenching of turbulent magnetic diffusivity has been recently suggested by Rogachevskii \& Kleeorin (2001) and used in a galactic dynamo model by Kleeorin et al. (2003).

We appreciate that alternative interpretations for the current helicity observations could be suggested, e.g. helicity could in principle be produced during active region formation rather than being connected with the magnetic field properties in the region of intensive dynamo action. A development of such an alternative explanation to the point where it could be confronted with the observational data looks highly desirable.

We conclude with the expression of a guarded but real optimism that magnetic helicity observations can result in the foreseeable future in a new level of understanding in dynamo theory, which will base the accepted $\alpha$-profiles not only on order-of-magnitude arguments and numerical simulations, but also on the observational data. It would be very important to support this progress in solar dynamo theory by similar progress in other areas of dynamo theory, in particular for galactic dynamos. We mention in this respect a recent suggestion of Enßlin \& Vogt (2003) concerning the possibility of observing the magnetic helicity of galactic magnetic fields.

Acknowledgements. We thank Axel Brandenburg for his comments on this paper. Financial support from NATO under grant PST.CLG 974737, RFBR under grants 03-02-16384, 01-02-17693, the RFBRNSFC grant 02-02-39027 and the INTAS Program Foundation under grants 99-348 and 2000-840 is acknowledged. DS and KK are grateful for support from the Chinese Academy of Sciences and NSFC towards their visits to Beijing.

\section{Appendix A: The nonlinear system of dynamo equations}

The system of nonlinear equations in nondimensional form for an axisymmetric mean magnetic field $\boldsymbol{B}=B \boldsymbol{e}_{\phi}+\boldsymbol{\nabla} \times\left(A \boldsymbol{e}_{\phi}\right)$ reads

$$
\begin{aligned}
& \frac{\partial B}{\partial t}=D \hat{\Omega} A+\Delta_{s} B \\
& \frac{\partial A}{\partial t}=\alpha B+\Delta_{s} A \\
& \frac{\partial \chi^{c}}{\partial t}+\frac{\chi^{c}}{T}=\left(\frac{2 R}{l}\right)^{2}\left(\sin ^{2} \theta\left(\nabla_{s} A\right)\left(\nabla_{s} B\right)-B \Delta_{s} A\right. \\
& \left.-\alpha B^{2}+\frac{C}{R} \nabla \cdot\left(l^{2} \Lambda_{\rho}^{-1} \chi^{v} \phi_{v} B^{2} \boldsymbol{e}_{r}\right)\right)+\kappa \Delta \chi^{c}
\end{aligned}
$$

where $\alpha=\chi^{v} \phi_{v}+\chi^{c} \phi_{m} / \rho(r), \Delta_{s}=\sin ^{2} \theta \nabla_{s}^{2} \equiv \Delta-1 /\left(r^{2} \sin ^{2} \theta\right)$, and

$$
\begin{aligned}
& B_{r}=\frac{R_{\alpha}}{r \sin \theta} \frac{\partial}{\partial \theta}(\sin \theta A), \quad B_{\theta}=-\frac{R_{\alpha}}{r} \frac{\partial}{\partial r}(r A), \\
& \nabla_{s} A=\frac{1}{r \sin \theta}\left(\boldsymbol{e}_{r} \frac{\partial}{\partial r}(r A)+\boldsymbol{e}_{\theta} \frac{1}{\sin \theta} \frac{\partial}{\partial \theta}(\sin \theta A)\right), \\
& \hat{\Omega} A=\frac{1}{r} \frac{\partial(\Omega, A r \sin \theta)}{\partial(r, \theta)}
\end{aligned}
$$

Now we consider dynamo action in a thin convective shell, average $A, B$ and $\alpha$ over the depth of the shell and consider these quantities as functions of colatitude $\theta$ only. Then we neglect the convection shell curvature and replace it by a flat slab. This implies that $\Delta_{s}=\Delta=\partial^{2} / \partial \theta^{2}-\mu^{2}, \sin \theta \boldsymbol{\nabla}_{s}=\boldsymbol{e}_{\theta}(\partial / \partial \theta)+\mu \boldsymbol{e}_{r}$ and $\hat{\Omega} A=g(\partial A / \partial \theta)$. We also redefine $C \mu l^{2} / \Lambda_{\rho} R$ as $C$. This yields Eqs. (5), (6) and (14).

\section{References}

Abramenko, V. I., Wang, T. J., \& Yurchishin, V. B. 1996, Sol. Phys., 168, 75

Abramenko, V. I., Wang, T. J., \& Yurchishin, V. B. 1997, Sol. Phys., 174, 291

Baker, N., \& Temesvary, S. 1966, Tables of Convective Stellar Envelope Models (New York)

Baliunas, S., Donahue, R. A., Soon, W. H., et al. 1995, ApJ, 438, 269 
Bao, S. D., Ai, G. X., \& Zhang, H. Q. 2002, in Highlights of Astronomy, V. 12, ed. H. Rickman (San Francisco) 392

Bao, S. D., Ai G. X., \& Zhang, H. Q. 2000, J. Astrophys. Astron., 21, 303

Bao, S. D., \& Zhang, H. Q. 1998, ApJ, 496, L43

Berger, M., \& Ruzmaikin, A. 2000, J. Geophys. Res., A105, 10481

Blackman, E. G., \& Brandenburg, A. 2002, ApJ, 579, 359

Blackman, E., \& Brandenburg, A. 2003, ApJ, 584, L99

Brandenburg, A., \& Dobler, W. 2001, A\&A, 369, 329

Brandenburg, A., Dobler, W., \& Subramanian, K. 2002, Astr. Nachr., 323, 99

Brandenburg, A., \& Sokoloff, D. 2002, GAFD, 96, 319

Brandenburg A., \& Subramanian, K. 2000, A\&A, 361, L33

Brooke, J., Moss, D., \& Phillips, A. 2002, A\&A, 395, 1013

Bruevich, E. A., Katsova, M. M., \& Sokoloff, D. D. 2001, Astron. Rep., 45, 718

Canfield, R. C., \& Pevtsov, A. A. 1998, in Synoptic Sol. Physics, ed. K. S. Balasubramaniamn, J. W. Harvey, \& D. M. Rabin, ASP Conf. Ser., 140, 131

Chae, J. 2001, ApJ, 560, L95

Covas, E., Tavakol, R., Tworkowski, A., \& Brandenburg, A. 1998, A\&A, 329, 350

Dikpati, M., \& Gilman, P. A. 2001, ApJ, 559, 428

Enßlin, T. A., \& Vogt, C. 2003, [astro-ph/0302426]

Field, G., Blackman, E., \& Chou, H. 1999, ApJ, 513, 638

Frisch, U., Pouquet, A., Leorat, I., \& Mazure, A. 1975, J. Fluid Mech., 68, 769

Galitski, V. M., \& Sokoloff, D. D. 1999, GAFD, 91, 147

Hoyt, D., Shatten, K., \& Nesme-Ribes, E. 1994, Geophys. Res. Lett., 21,2067

Iroshnikov, R. S. 1970, SvA, 14, 582; 1001

Kleeorin, N., Moss, D., Rogachevskii, I., \& Sokoloff, D. 2000, A\&A, 361, L5

Kleeorin, N., Moss, D., Rogachevskii, I., \& Sokoloff, D. 2002, A\&A, 387,453

Kleeorin, N., Moss, D., Rogachevskii, I., \& Sokoloff, D. 2003, A\&A, 400,9

Kleeorin, N., \& Rogachevskii, I. 1999, Phys. Rev. E., 59, 6724

Kleeorin, N., Rogachevskii, I., \& Ruzmaikin, A. 1994, Sol. Phys., 155, 223
Kleeorin, N., Rogachevskii, I., \& Ruzmaikin, A. 1995, A\&A, 297, 159

Kleeorin, N., \& Ruzmaikin, A. 1982, Magnetohydrodynamics, 18, 116

Krause, F., \& Rädler, K.-H. 1980, Mean-Field Magnetohydrodynamics (Oxford: Pergamon)

Kuzanyan, K. M., \& Sokoloff, D. 1995, GAFD, 81, 113

Kuzanyan, K. M., Bao S., \& Zhang, H. 2000, Sol. Phys., 191, 231

Longcope, D. W., Fisher, G. H., \& Pevtsov, A. A. 1998, ApJ, 507, 417

Moffatt, H. K. 1978, Magnetic Field Generation in Electrically Conducting Fluids (New York: Cambridge Univ Press)

Parker, E. 1955, ApJ, 121, 491

Parker, E. 1979, Cosmical Magnetic Fields. (Oxford: Clarendon)

Pevtsov, A. A., Canfield, R. C., \& Metchalf, T. R. 1994, ApJ, 425, L117

Pevtsov, A. A., Canfield, R. C., \& Metchalf, T. R. 1995, ApJ, 440, L109

Pevtsov, A. A., Canfield, R. C., \& Latushko, S. M. 2001, ApJ, 549, L261

Rogachevskii, I., \& Kleeorin, N. 2000, Phys. Rev. E, 61, 5202

Rogachevskii, I., \& Kleeorin, N. 2001, Phys. Rev. E, 64, 056307

Rust, D. M., \& Kumar, A. 1996, ApJ, 464, L119

Rüdiger, G., \& Brandenburg, A. 1995, A\&A, 296, 557

Seehafer, N. 1990, Sol. Phys., 125219

Sofia, S., Fox, P., \& Schatten, K. 1998, Geophys. Res. Lett., 25, 4149

Sokoloff, D., Fioc, M., \& Nesme-Ribes, E. 1995, Magnetohydrodynamics, 31,18

Spruit, H. C. 1974, Sol. Phys. 34, 277

Tavakol, R., Covas, E., Moss, D., \& Tworkowski, A. 2002, A\&A, 389, 1100

Wang, T. J., Ai, G. X., \& Deng, Y. Y. 1996, Astrophys. Rep. (Publ. Beijing Obs.), 28, 31

Woltjer, L. 1958, Proc. Nat. Acad. Sc. USA, 44489

Zhang, H., \& Bao, S. 1998, A\&A, 339, 880

Zhang, H., \& Bao, S. 1999, ApJ, 519, 876

Zhang, H., Bao, S., \& Kuzanyan, K. M. 2002, Astron Rep., 46, 414

Zeldovich, Ya. B., Ruzmaikin, A. A., \& Sokoloff, D. D. 1983, Magnetic Fields in Astrophysics (New York: Gordon and Breach) 